\title{
Detecting chronic apical periodontitis for improved endodontic success
}

2018 Theme Article: Oral manifestations of systemic disease

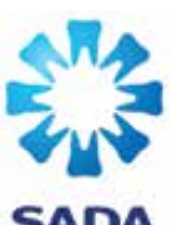

SADJ June 2018, Vol 73 no 5 p359 - p361

NA Fernandes', GO Patterson ${ }^{2}$, LM Sykes ${ }^{3}$

\section{SUMMARY}

Endodontic treatments are frequently carried out in daily clinical practice. The presence or absence of a periapical radiolucency, assessed using intraoral radiographs, is one of the criteria used to determine endodontic success. When such a lesion is present around the apex of a tooth, the condition is known as chronic apical periodontitis. While this is common around root treated teeth, it can also be associated with otherwise healthy teeth. In both instances, it represents an inflammatory response to bacteria or irritants in the periapical space, and results in bone loss and areas of reduced density, which can then form apical granulomas or cysts. The literature has shown that periapical lesions can go undetected, due to the two-dimensional limitations of periapical radiographs. These "hidden" lesions can compromise the longevity of the tooth. Considering that apical periodontitis can be far more accurately detected and diagnosed on cone beam computed tomography, it has been recommended by several authors that endodontic treatment outcomes ideally should be evaluated using this imaging modality, whenever possible.

\section{INTRODUCTION}

Endodontic success has largely been based on three basic principles known as the "endodontic triad of success" which includes: cleaning (debridement and disinfection), shaping, and obturation (sealing). ${ }^{1}$ It is believed that if all of these are carried out meticulously, then the treatment will be effective. ${ }^{2}$ However, many clinicians can attest to the fact that endodontic failures occur despite their strict adherence to these principles. Instrumentation and antibacterial irrigation with sodium hypochlorite will render around only $50 \%$ of canals to be microbialfree. Any remaining canals will contain small numbers

1. Nelson A Fernandes: BDS. Department of Prosthodontics, School of Dentistry, Faculty of Health Sciences, University of Pretoria, South Africa.

2. Go Patterson: $B C h D, M C h D(O M P)$. Netcare Pretoria East Hospital, Pretoria, South Africa.

3. Leanne M Sykes: BSC, BDS, MDent (Pros). Department of Prosthodontics, School of Dentistry, Faculty of Health Sciences, University of Pretoria, South Africa.

\section{Corresponding author}

\section{Nelson A Fernandes:}

Department of Prosthodontics, School of Dentistry, Faculty of Health Sciences, University of Pretoria, South Africa. PO Box 1266, Pretoria 0001, South Africa. Tel: 012319 2388. E-mail: nelson.fernandes@up.ac.za

\author{
ACRONYMS \\ CAP: chronic apical periodontitis \\ CBCT: cone beam computed tomography \\ CT: computed tomography \\ ETT: endodontically treated teeth \\ FOV: field of view \\ PAI: periapical index \\ PDL: periodontal ligament \\ PR: periapical radiographs
}

of recoverable bacteria, and these are eliminated only if antimicrobial dressings are applied to the canals before obturation. ${ }^{3}$ Possible causes of these failures could be related to the anatomical complexity of root canal systems, creating areas that cannot be cleaned and obturated adequately, missed canals, and even the composition of any residential bacterial in canal systems. Studies have also shown that the microorganism Enterococcus faecalis is resistant to intracanal medicaments and is then a possible cause of endodontic failure. ${ }^{4}$ Some failures become more perplexing when seen alongside other cases that have succeeded despite comparatively substandard treatment. Believing that these opposing scenarios can occur simultaneously is referred to as cognitive dissonance and poses a dilemma for the clinician. ${ }^{2}$ One possible explanation could be that many clinicians do not pay enough attention to the evaluation of periapical regions surrounding endodontically treated teeth (ETT).2,5,6

\section{APICAL PERIODONTITIS}

Patient-reported symptoms following endodontic therapy are very subjective and variable. Clinicians often judge success by assessing the status of the periapical tissues surrounding the tooth in question, using periapical radiographs (PR) to detect the presence (or absence) of radiolucencies in this region. ${ }^{6-11}$ When a radiolucency is present, the tooth is diagnosed as having apical periodontitis, which is an inflammatory response to bacterial infection and irritants within the root canal system, ${ }^{8}$ and may be referred to as chronic apical periodontitis (CAP) for the response develops over a long time. Considering that these lesions are often associated with ETT, ${ }^{6-11}$ they are generally used as a criterion in the 
assessment of endodontic success or failure. ${ }^{12}$ Rarely, such lesions may also occur in teeth that have not been endodontically treated, such as those subjected to occlusal trauma, and teeth presenting with periapical cemento-osseous dysplasia or benign cysts. ${ }^{12-15}$

CAP involves a host inflammatory response that develops over time, resulting in structural bone changes (bone resorption) and granulation tissue formation, which are characteristic of chronic inflammation. ${ }^{12,16,17}$ The quality of the endodontic treatment affects the extent of the lesion. While it is agreed that the technical quality of root obturation has a significant impact on the presence or absence of CAP, endodontic success can be achieved only if canal disinfection and coronal sealing have also been adequately performed. ${ }^{8,11}$ This is because the latter procedures directly influence the introduction of bacteria into the periapical region, or the persistence of bacteria arising from the treated canals. ${ }^{11,12}$ There are five biological factors which may cause a chronic periapical radiolucency following endodontic therapy. These include: intraradicular infection in the apical root canal system; extraradicular infection due to actinomycosis; cystic lesions; foreign body reactions (endogenous and exogenous), and scar-tissue healing of the lesion. ${ }^{4}$ However, persistent microbial infection in the apical portion of completed endodontic therapies remains the major cause of CAP. ${ }^{4}$ Accepting that final confirmation of the diagnosis of CAP can be made only with a histopathological examination, several researchers have conducted studies in the endeavour to correlate the radiographic appearance of CAP with associated histological findings. ${ }^{14}$ Histologically, these tissues all contain a variable chronic inflammatory cell infiltrate composed of macrophages, lymphocytes, and plasma cells, surrounded by a fibrous capsule. Depending on their contents, these lesions are referred to either as periapical granulomas or radicular cysts. ${ }^{18}$ Making this distinction is important, as it impacts on the type of treatment (surgical or non-surgical) required for resolution of the lesion.

\section{RADIOGRAPHIC FINDINGS}

Radiographically, CAP is diagnosed when there is a radiolucency in the apical part of a root which is at least twice the width of the normal periodontal ligament (PDL) space. ${ }^{6,8}$ To aid in diagnosing these lesions, an index known as the periapical index (PAl), was developed over 30 years ago, ${ }^{14}$ which quantified the extent of these lesions. This index has been used in several epidemiological studies, and was previously accepted as the gold standard for assessing treatment outcome following endodontic therapy. ${ }^{15}$ PAl quantified periapical inflammation via a scoring system (Table 1), from 1-5, ranging from no disease (healthy periapical region) to severe periodontitis. ${ }^{12,17}$ However, this index was based on a single study of correlations between radiographic

\begin{tabular}{|c|c|}
\hline Score & Description \\
\hline 1 & Normal periodontium apically \\
\hline 2 & Small changes in bone structure (apical periodontitis) \\
\hline 3 & Structural bone changes with some mineral loss \\
\hline 4 & Periodontitis with a well-defined radiolucency \\
\hline 5 & Radiolucency with radiating bone expansion \\
\hline
\end{tabular}

appearance and histologic findings, using only anterior maxillary teeth. This raised concerns regarding its validity in assessing the periapical health for all teeth wherever positioned in the mouth. ${ }^{8,15}$ These misgivings are due to the fact that conventional PR are not very sensitive or specific, and though the radiograph may be useful in the detection of disease to varying degrees when radiolucencies are present, the lack of such features is not always indicative of periapical health. ${ }^{6}$ Another major limitation is that PR are two-dimensional (2D) representations of three-dimensional (3D) structures, and are thus prone to distortion, as a result of which several clinical features may not be visible.15-17 For example, bony lesions present within the cancellous bone may be obscured by an overlying thicker cortex, and go undetected. This lack of sensitivity is compounded by the fact that $30-50 \%$ of bone mineral loss is required before radiolucencies become apparent radiographically. ${ }^{16-18}$ Radiographic interpretation is also sensitive to small changes in the angulation of the $\mathrm{x}$-ray tube-head which can severely affect the size of the images. ${ }^{7}$ The surrounding bone density also plays a role, and lesions are more easily detected in areas of reduced bone density (anterior maxilla), when compared with denser areas (posterior mandible)..$^{17}$ Relying on conventional radiographs in assessing the periapical status of teeth may be problematic, as the probability of inaccurate diagnoses is high. ${ }^{16}$

\section{CONE BEAM COMPUTED TOMOGRAPHY}

Cone beam computed tomography (CBCT) is a $3 D$ imaging system which was specifically developed for the maxillo-facial region. ${ }^{20} \mathrm{CBCT}$ scans allow for multi-planar assessment of oral structures, that is in the transverse (axial), frontal (coronal), and cross-sectional (sagittal) planes, at significantly lower radiation doses than those delivered by medical computed tomography (CT). ${ }^{21} \mathrm{~A}$ cone-shaped beam of radiation captures the entire 3D volume of the required area of investigation, known as the field of view (FOV), in a single rotation of the CBCT scanner. As the entire volume is captured at the same time, a 3D assessment of the area of interest can be performed at a sub-millimeter spatial resolution. ${ }^{20}$ This technology is becoming ever more prevalent in Dentistry, with several recognized endodontic applications already in use. These include: the assessment of root canal anatomy (eg. accessory canals such as MB2), root fractures, root resorption, periapical pathology, and pre-operative planning of apical surgery (apicoectomy). ${ }^{17}$

Numerous studies have found that CBCT is considerably more accurate in assessing the periapical regions surrounding teeth than are conventional periapical radiographs. ${ }^{16,20}$ Results showed that as many as $30-40 \%$ of CAP lesions diagnosed on CBCT, were not visible on PR. CBCT has also been shown to be a reliable method of distinguishing between solid (granulomas), and fluid-filled (cystic) lesions, as determined by differences in grayscale values which can be used to measure density. This distinction was previously possible only on histological examination. Thus CBCT offers a more rapid and non-invasive means of diagnosis. ${ }^{17}$

CBCT has also been shown to be more accurate in determining periapical health following endodontic treatment. Teeth showing reduced periapical radiolucencies on PR were often diagnosed as resolving, yet have been found to have 
enlarged lesions on CBCT. This occurs if there has been an expansion of a lesion confined to the cancellous bone, whereby its volumetric expansion can only be detected on $3 \mathrm{D}$ imaging, such as $\mathrm{CBCT} .^{15}$ Thus, what appears to be endodontic success and healing on PR, may be in fact, failure, as evidenced by CBCT. While the advantage of limiting the FOV to 3-4 individual teeth significantly reduces radiation doses, they are still considerably higher than those of conventional radiographs. ${ }^{6}$ In addition, high costs, limited availability of $\mathrm{CBCT}$, and increased radiation dosage may preclude its routine use.

Complete radiographic resolution of CAP seldom occurs, but this factor alone should not define endodontic success or failure. Correlation between radiographic findings and clinical symptoms should always be made and if previous radiolucent areas become arrested, with no associated pain, loss of function, or surrounding tissue destruction, then the treatment can be regarded as being successful. However, radiographic evidence of an expanding lesion, causing bone resorption, should be considered as failure even in the absence of clinical symptoms, and should be further evaluated on CBCT imaging. Indeed, there may be cause to further investigate previously reported systematic reviews of endodontic success rates, whose findings were based on PR alone.

As the number of CBCT scans being taken on patients is increasing, so too are more endodontic failures being noticed, many of which are a result of undiagnosed CAP. If one considers that most post-endodontic complications usually occur within the first six months to two years after completion of treatment, patients should routinely be recalled during this time to assess the periapical status of root treated teeth. If failures are suspected or detected, then decisive interventions can be undertaken at an early stage rather than waiting longer periods. Prolonged waiting times, in the hope that a "questionable" tooth will eventually heal itself, should be avoided as this allows long-standing infections to cause significant amounts of bone loss/resorption (Figure 1). ${ }^{7,15,18}$

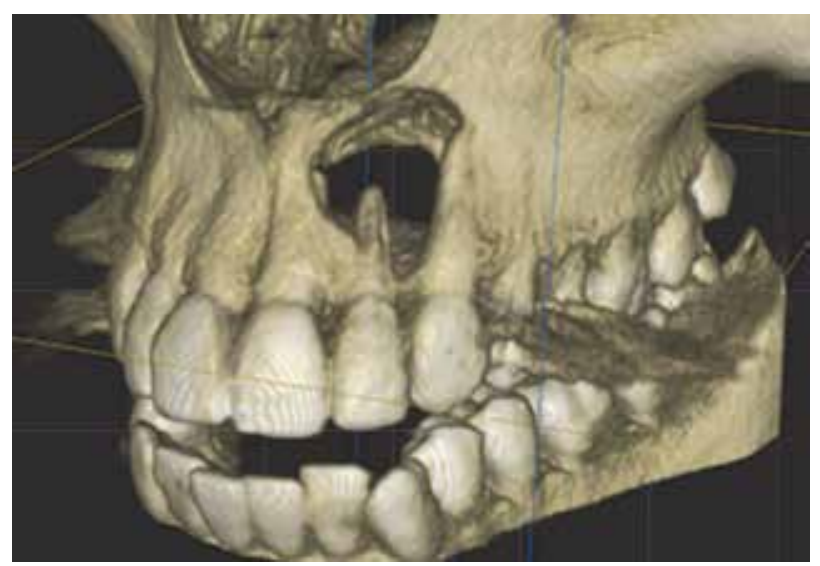

Figure 1: 3D volumetric reconstruction of a CBCT scan taken on a patient referred for apical surgery (root-end resection) on tooth 22. Note the extensive bone destruction that has taken place because of failed endodontics.

\section{CONCLUSION}

CAP is commonly found associated with endodontically treated teeth. Inadequate obturation significantly increases the risk of CAP. This often goes undetected on PR, but is seen with increasing frequency on CBCT scans. Though other factors may contribute to the development of CAP around a tooth, inadequate endodontic therapy leading to persistent intra-radicular microbial infection is by far the most common cause. Failure to detect and to intervene timeously to remediate endodontic failures may radically compromise the survival of teeth and limit future treatment options due to the associated bone destruction and loss.

\section{References}

1. Ingle Jl. A standardized endodontic technique utilizing newly designed instruments and filling materials. Oral Surg Oral Med Oral Pathol. 1961; 14(1): 83-91.

2. Seltzer S, Bender I. Cognitive dissonance in endodontics. Oral Surg Oral Med Oral Pathol. 1965; 20(4): 506-16.

3. Nair P. Pathogenesis of apical periodontitis and the causes of endodontic failure. Crit Rev Oral Biol Med. 2004; 15(6): 348-381.

4. Estrela C, Holland R, de Araujo Estrela CR, Alencar AH, Sousa-Neto MD, Pecora JD. Characterisation of successful root canal treatment. Braz Dent J. 2014; 24(5): 3-11.

5. Hoskinson SE, Ng Y-L, Hoskinson AE, Moles DR, Gulabivala K. A retrospective comparison of outcome of root canal treatment using two different protocols. Oral Surg Oral Med Oral Pathol Oral Radiol Endod. 2002; 93(6): 705-15.

6. Kanagasingam S, Mannocci F, Lim CX, Yong CP, Patel S. Diagnostic accuracy of periapical radiography and cone beam computed tomography in detecting apical periodontitis using histopathological findings as a reference standard. Int Endod J. 2016 ;50(6):417-26.

7. Bender IB, Seltzer S, Soltanoff W. Endodontic success- a reappraisal of criteria. Part I. Oral Surg Oral Med Oral Pathol. 1966; 22(6): 780-9.

8. Van der Veken D, Curvers F, Fieuws S, Lambrechts P. Prevalence of apical periodontitis and root filled teeth in a Belgian subpopulation found on CBCT images. Int Endod J. 2016; 50(4):317-29.

9. Patel S, Mannocci F, Shemesh H, Wu MK, Wesselink PR, Lambrechts P. Radiographs and CBCT- time for a reassessment? Int Endod J. 2011; 44(10): 887-8.

10. Christiansen R, Kirkevang LL, Gotfredsen E, Wenzel A. Periapical radiography and cone beam computed tomography for assessment of the periapical bone defect 1 week and 12 months after root-end resection. Dentomaxillofac Radiol. 2009; 38(8): 531-6.

11. Huumonen S, Suominen AL, Vehkalahti MM. Prevalence of apical periodontitis in root filled teeth: findings from a nationwide survey in Finland. Int Endod J. 2017; 50(3): 229-36.

12. Ørstavik D. Time-course and risk analysis of the development and healing of chronic apical periodontitis in man. Int Endod J. 1996; 29(3): 150-5.

13. Orstavik D. Reliability of the periapical index scoring system. Scand J Dent Res. 1988; 96(2): 108-11.

14. Ørstavik D, Kerekes K, Eriksen HM. The periapical index: A scoring system for radiographic assessment of apical periodontitis. Endod Dent Traumatol. 1986; 2(1): 20-34.

15. Wu MK, Shemesh H, Wesselink PR. Limitations of previously published systematic reviews evaluating the outcome of endodontic treatment. Int Endod J. 2009; 42(8): 656-66.

16. Estrela C, Bueno MR, Leles CR, Azevedo B, Azevedo JR. Accuracy of cone beam computed tomography and panoramic and periapical radiography for detection of apical periodontitis. J Endod. 2008; 34(3): 273-9.

17. Estrela C, Bueno MR, Azevedo BC, Azevedo JR, Pecora JD. A new periapical index based on cone beam computed tomography. J Endod. 2008; 34(11): 1325-31.

18. Bender IB, Seltzer S, Soltanoff W. Endodontic success- a reappraisal of criteria. Part II. Oral Surg Oral Med Oral Pathol. 1966; 22(6): 790-802.

19. Parker J, Mol A, Rivera EM, Tawil P. CBCT uses in clinica endodontics: the effect of CBCT on the ability to locate MB2 canals in maxillary molars. Int Endod J. 2016;50(12):1109-15.

20. Patel S, Dawood A, Pitt Ford T, Whaites E. The potential applications of cone beam computed tomography in the management of endodontic problems. Int Endod J. 2007; 40(10): 818-30.

21. Patel S. New dimensions in endodontic imaging: Part 2. Cone beam computed tomography. Int Endod J. 2009; 42(6): 463-75. 